\title{
Holographic complexity: stretching the horizon of an evaporating black hole
}

\author{
Lukas Schneiderbauer, Watse Sybesma and Lárus Thorlacius \\ Science Institute, University of Iceland, \\ Dunhaga 3, Reykjavík 10\%, Iceland \\ E-mail: lukas.schneiderbauer@gmail.com, watse@hi.is, lth@hi.is
}

ABSTRACT: We obtain the holographic complexity of an evaporating black hole in the semi-classical RST model of two-dimensional dilaton gravity, using a volume prescription that takes into account the higher-dimensional origin of the model. For classical black holes, we recover the expected late time behaviour of the complexity, but new features arise at the semi-classical level. By considering the volume inside the stretched horizon of the evolving black hole, we obtain sensible results for the rate of growth of the complexity, with an early onset of order the black hole scrambling time followed by an extended period where the rate of growth tracks the shrinking area of the stretched horizon as the black hole evaporates.

KEYwords: 2D Gravity, Black Holes, Classical Theories of Gravity

ARXiv EPrint: 1911.06800 


\section{Contents}

1 Introduction 1

2 Complexity of classical CGHS black holes 2

2.1 Complexity of a two-sided black hole 3

$\begin{array}{lll}2.2 & \text { Complexity of a black hole formed by gravitational collapse } & 7\end{array}$

3 The RST model: complexity in a semi-classical black hole 9

4 Conclusion and outlook $\quad 12$

\section{Introduction}

The interior of a black hole is the archetype of an emergent spacetime in the holographic approach to quantum gravity. The principle of black hole complementarity posits that the interior geometry and any matter that enters a black hole can be described in terms of a finite number of quantum mechanical degrees of freedom associated with a stretched horizon located outside the event horizon [1]. In order to reproduce black hole thermodynamics, the number of stretched horizon degrees of freedom should match the Bekenstein-Hawking entropy and the dynamics must be sufficiently chaotic to scramble quantum information on a relatively short timescale, but, beyond that, the precise nature of the stretched horizon dynamics and the holographic encoding of the black hole interior remain elusive. In what follows, we will not make any specific assumptions about the scrambling dynamics but it can be useful to keep in mind a collection of qubits undergoing k-local interactions as a simple model [2].

Quantum complexity has in recent years emerged as an important entry in the holographic dictionary for black holes following Susskind's conjecture that the expanding spatial volume of the Einstein-Rosen bridge of a two-sided eternal black hole reflects the growing complexity of a corresponding quantum state [3]. In the present paper, we explore the relation between quantum complexity and interior black hole geometry in the context of semi-classical black holes that are formed by gravitational collapse and subsequently evaporate due to the emission of Hawking radiation. Our main result, based on explicit calculations in a two-dimensional dilaton gravity model that allows analytic study of semiclassical effects, is that the rate of growth of holographic complexity precisely tracks the shrinking area of the stretched horizon as the black hole evaporates, where the stretched horizon is taken to be a membrane with an area larger than that of the event horizon by order one in the appropriate units of the model.

The dilaton gravity model has explicit classical solutions describing black hole formation from arbitrary incoming matter energy flux. We focus, for simplicity, on black holes 
formed by an infalling thin shell and start off by adapting the complexity as volume conjecture to this context. A suitably defined volume functional exhibits precisely the expected linear growth with time at late times and by restricting to the volume inside the stretched horizon of the dynamically formed black hole one finds reasonable early-time behaviour as well. We then consider a semi-classical extension of the model where the field equations remain analytically soluble and numerically evaluate the volume functional in an evaporating black hole background.

The transitory nature of semi-classical black holes highlights certain technical aspects of the identification between complexity and volume, that can often be ignored when considering classical black holes. In the present paper, we only consider the volume representation of the semi-classical black hole complexity, where these issues are relatively easy to address. The alternative formulation of holographic complexity in terms of the action on a Wheeler-DeWitt patch $[4,5]$ is also of interest for these dilaton gravity models, but it is more subtle to implement at the semi-classical level, and we postpone this to a forthcoming paper [6].

\section{Complexity of classical CGHS black holes}

We work within a class of two-dimensional dilaton gravity theories first introduced by Callan, Giddings, Harvey, and Strominger (CGHS) [7]. These are simple toy theories for black hole physics that can be systematically studied at the semi-classical level. They have classical solutions that describe black hole geometries with a spacelike singularity inside an event horizon. The black holes include static two-sided black holes and also dynamical black holes formed by the gravitational collapse of matter fields. The quantization of matter fields in a black hole background leads to Hawking radiation and its back-reaction on the geometry causes the black hole to evaporate. The subsequent evolution is particularly simple to track in a variant of the semi-classical model that was introduced by Russo, Susskind, and Thorlacius (RST), where the semi-classical field equations can be solved analytically [8].

The original CGHS model can be viewed as a spherical reduction of a four-dimensional dilaton gravity theory in a near-extremal magnetically charged black hole background $[9$, 10]. The two-dimensional theory captures the low-energy dynamics of radial modes in the near-horizon region of higher-dimensional geometry. The volume that is to be identified as the quantum complexity is that of a spacelike three-dimensional surface in the original theory, rather than the length of a spacelike curve in two-dimensions, and this will be reflected in our calculations below.

The CGHS model and related semi-classical models were studied extensively in the early 1990's and several reviews were written at that time, including [11-14]. We will be brief and only introduce the minimal ingredients needed for the purposes of this paper.

The classical GGHS action,

$$
S_{\mathrm{CGHS}}=\int_{\mathcal{M}} d^{2} x \sqrt{-g}\left[e^{-2 \phi}\left(R+4(\nabla \phi)^{2}+4 \lambda^{2}\right)-\frac{1}{2} \sum_{i=1}^{N}\left(\nabla f_{i}\right)^{2}\right],
$$


involves the two-dimensional metric, a scalar dilaton field, and matter in the form of $N$ minimally coupled scalar fields $f_{i}$. The two-dimensional theory inherits a scale $\lambda$ from the parent theory set by the magnetic charge of the near-extremal black hole. In the following, we take length to be measured in units of $\lambda^{-1}$ and thus set $\lambda=1$.

We find it convenient to work in a conformal gauge,

$$
d s^{2}=-e^{2 \rho} d x^{+} d x^{-},
$$

and use a residual conformal reparametrisation to choose coordinates where $\rho=\phi$. These are referred to as Kruskal coordinates for reasons that will become apparent below. In this coordinate system, the classical equations of motion and constraints reduce to

$$
\partial_{+} \partial_{-} f_{i}=0, \quad \partial_{+} \partial_{-} e^{-2 \phi}=-1, \quad \partial_{ \pm}^{2} e^{-2 \phi}=-T_{ \pm \pm}^{f},
$$

where $T_{\mu \nu}^{f}$ is the energy-momentum tensor of the $f_{i}$ matter fields.

In order to obtain the holographic complexity of a classical CGHS black hole, we introduce a volume functional

$$
V=\int d s e^{-2 \phi} \sqrt{g_{\mu \nu} \dot{y}^{\mu} \dot{y}^{\nu}}
$$

where $y^{\mu}(s)$ is a spacelike curve in the two-dimensional spacetime and the integrand includes a factor of $e^{-2 \phi}$, which is proportional to the area of the local transverse two-sphere $S^{2}$ of the near-extremal dilaton black hole (in Einstein frame) in the higher-dimensional parent theory. A corresponding factor was included when defining $\mathcal{C}_{V}$ for black holes in two-dimensional Jackiw-Teitelboim gravity in [15]. In the case of the Jackiw-Teitelboim black hole, the transverse $S^{2}$ has constant area and the calculation of the complexity reduces to calculating a two-dimensional geodesic length. For a CGHS black hole, on the other hand, the transverse area depends on spatial location and curves that maximize (2.4) are not geodesics.

\subsection{Complexity of a two-sided black hole}

The first solution we consider describes a two-sided eternal black hole,

$$
e^{-2 \phi}=e^{-2 \rho}=M-x^{+} x^{-}, \quad f_{i}=0 .
$$

From the Ricci scalar,

$$
R=-2 \nabla^{2} \rho=\frac{4 M}{M-x^{+} x^{-}},
$$

it is apparent that, for $M>0$, the curvature is singular on the spacelike curves $x^{+} x^{-}=M$, corresponding to the white hole and black hole singularity. The Penrose diagram, shown in figure 1, is identical to the one obtained for a Schwarzschild black hole in $3+1$ dimensional Einstein gravity. The event horizon is at $x^{+} x^{-}=0$ and there are two asymptotic regions, $-x^{+} x^{-} \rightarrow \infty$, where the curvature goes to zero. One can introduce Schwarzschild-like coordinates $(t, \sigma)$ in the outside region on the right (where $x^{+}>0$ and $x^{-}<0$ ) via the transformation $x^{ \pm}= \pm e^{ \pm t+\sigma}$. The corresponding coordinate transformation on the left 


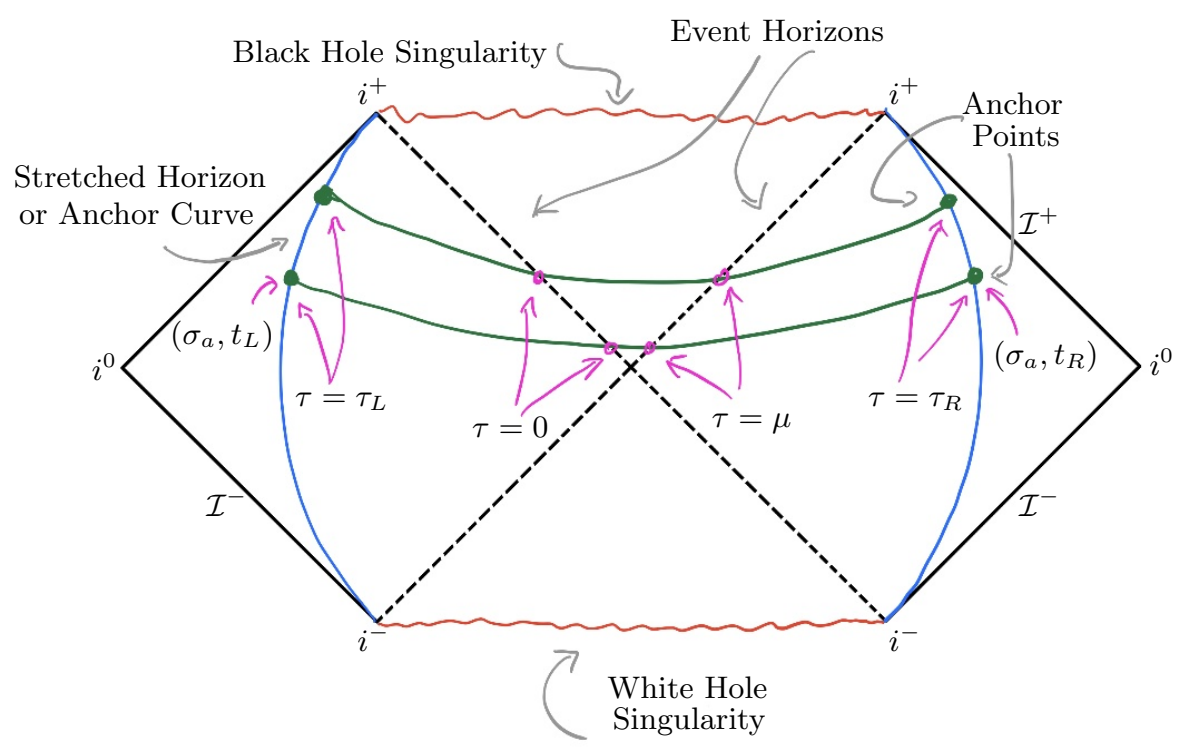

Figure 1. This cartoon depicts the Penrose diagram of a two-sided eternal CGHS black hole.

(where $x^{+}<0$ and $x^{-}>0$ ) is given by $x^{ \pm}=\mp e^{\mp t+\sigma}$. With these conventions, the metric in $(t, \sigma)$ coordinates approaches the two-dimensional Minkowski metric as $\sigma \rightarrow+\infty$ on both sides of the black hole and $t$ propagates to the future in the 'upward' direction on both sides.

The volume in (2.4) is divergent for spacelike curves that extend all the way to spatial infinity. In order to obtain a finite expression for the complexity, we introduce timelike anchor curves outside the black hole where the volume integral is cut off (see figure 1). We find it convenient to use anchor curves on which the dilaton field is constant, $\phi\left(x_{a}^{+}, x_{a}^{-}\right)=$ $\phi_{a}$, providing a coordinate invariant notion of spatial position outside the black hole, and place the curves symmetrically on the left- and right-hand side of the black hole. The anchor curves take a particularly simple form in the Schwarzschild-like coordinates, where they are curves of constant $\sigma=\sigma_{a}$, with

$$
\sigma_{a}=\frac{1}{2} \log \left(e^{-2 \phi_{a}}-M\right)
$$

where the anchor curve on the left (right) is parametrised by $t_{L}\left(t_{R}\right)$.

Our prescription for the volume complexity $\mathcal{C}_{V}\left(t_{R}, t_{L}\right)$ of a two-sided CGHS black hole is then given by the maximal volume on the set of spacelike curves $\left(y^{+}(s), y^{-}(s)\right)$ with fixed endpoints at $t_{L}$ and $t_{R}$ on the left and right anchor curves. This amounts to maximizing the volume functional,

$$
V=\int d s \sqrt{-\dot{y}^{+} \dot{y}^{-}\left(M-y^{+} y^{-}\right)},
$$

and evaluating the resulting maximal volume.

In order to proceed, we note that the functional is invariant under the transformations

$$
\begin{aligned}
& y^{+} \mapsto e^{\epsilon} y^{+}, \\
& y^{-} \mapsto e^{-\epsilon} y^{-},
\end{aligned}
$$


for $\epsilon \in \mathbb{R}$. The corresponding conserved quantity is given by

$$
E=\sqrt{\frac{M-y^{+} y^{-}}{-\dot{y}^{+} \dot{y}^{-}}}\left(\dot{y}^{+} y^{-}-y^{+} \dot{y}^{-}\right) .
$$

We construct the corresponding maximum volume curve by first focusing on the outside region on the right and rewriting the conserved charge in the $(t, \sigma)$ coordinates,

$$
E=-2 e^{\sigma} \frac{d t}{d \sigma} \sqrt{\frac{M+e^{2 \sigma}}{1-\left(\frac{d t}{d \sigma}\right)^{2}}}
$$

This integrates to

$$
t-t_{0}=-\sigma+\frac{1}{2} \log \left(E^{2}+2 M e^{2 \sigma}+E \sqrt{E^{2}+4 M e^{2 \sigma}+4 e^{4 \sigma}}\right) .
$$

We then convert back to Kruskal coordinates by using $y^{ \pm}= \pm e^{ \pm t+\sigma}$ and obtain the following equation for a maximal volume curve, which is valid over the entire extended spacetime,

$$
e^{-2 t_{0}}\left(y^{+}\right)^{2}+4 M y^{+} y^{-}+4 e^{2 t_{0}}\left(M^{2}-E^{2}\right)\left(y^{-}\right)^{2}-2 E^{2}=0 .
$$

Maximal volume curves that extend between the anchor curves correspond to a conserved charge in the range $-M<E<M$, and can be parametrised as

$$
y^{+}=\sqrt{2} e^{t_{0}} \epsilon \sinh \tau, \quad y^{-}=-\frac{e^{-t_{0}}}{\sqrt{2}} \sinh (\tau-\mu),
$$

with $\epsilon=\sqrt{M^{2}-E^{2}}$ and $\tanh \mu=\frac{E}{M}$, while curves satisfying (2.13) with $|E|>M$ run into the curvature singularity. The parameter $\tau$ in (2.14) runs from a negative value $\tau_{L}<0$ at the endpoint on the left anchor curve to $\tau=0$, where the curve enters the black hole from the left. At $\tau=\mu$ the curve exits the black hole to the right and reaches the endpoint on the right anchor curve at $\tau=\tau_{R}$. We illustrate this setup in figure 1 .

The maximal volume curve is labelled by $E$ and $t_{0}$ in (2.14) but these labels are in oneto-one correspondence with the Schwarzschild times $t_{L}$ and $t_{R}$ where the curve meets the anchor curves. To see this, consider the intersection points between the maximal volume curve and the anchor curves. On the one hand we have

$$
e^{2 \sigma_{a}}=\epsilon \sinh \tau_{R} \sinh \left(\tau_{R}-\mu\right)=\epsilon \sinh \left(-\tau_{L}\right) \sinh \left(\mu-\tau_{L}\right)
$$

relating curve parameters to the spatial location of the anchor curves, and on the other hand a pair of relations involving the Schwarzschild times at the anchor points,

$$
e^{2 t_{R}}=2 \epsilon e^{2 t_{0}} \frac{\sinh \left(\tau_{R}\right)}{\sinh \left(\tau_{R}-\mu\right)}, \quad e^{2 t_{L}}=\frac{e^{-2 t_{0}}}{2 \epsilon} \frac{\sinh \left(\mu-\tau_{L}\right)}{\sinh \left(-\tau_{L}\right)} .
$$

The second equation in (2.15) is satisfied by imposing $\tau_{R}=\mu-\tau_{L}$ and the time relations can then be re-expressed as

$$
e^{t_{R}-t_{L}}=2 \epsilon e^{2 t_{0}}, \quad e^{t_{R}+t_{L}}=\frac{\sinh \left(\tau_{R}\right)}{\sinh \left(\tau_{R}-\mu\right)} .
$$


By combining (2.15) with the second equation in (2.17) and doing some algebra one eventually arrives at

$$
\epsilon=-e^{2 \sigma_{a}} \cosh \left(t_{R}+t_{L}\right)+\sqrt{e^{4 \sigma_{a}} \cosh ^{2}\left(t_{R}+t_{L}\right)+2 M e^{2 \sigma_{a}}+M^{2}} .
$$

The parametrisation (2.14) can then be re-expressed in terms of $t_{R}$ and $t_{L}$, as

$$
y^{+}=e^{\frac{1}{2}\left(t_{R}-t_{L}\right)} \sqrt{\epsilon} \sinh \tau, \quad y^{-}=-e^{-\frac{1}{2}\left(t_{R}-t_{L}\right)} \sqrt{\epsilon} \sinh (\tau-\mu) .
$$

with $\epsilon\left(t_{R}+t_{L}\right)$ given by (2.18).

The volume functional (2.8) is easily evaluated in this parametrisation,

$$
\begin{aligned}
V & =\epsilon \int_{\tau_{L}}^{\tau_{R}} d \tau \cosh \tau \cosh (\tau-\mu) \\
& =\frac{M}{2}\left(2 \tau_{R}-\mu\right)+\frac{\epsilon}{2} \sinh \left(2 \tau_{R}-\mu\right) .
\end{aligned}
$$

The first equation in (2.15) can be combined with second equation in (2.17) to give

$$
2 \tau_{R}-\mu=\operatorname{arccosh}\left[\frac{1}{M}\left(e^{2 \sigma_{a}} \cosh \left(t_{R}+t_{L}\right)+\sqrt{e^{4 \sigma_{a}} \cosh ^{2}\left(t_{R}+t_{L}\right)+2 M e^{2 \sigma_{a}}+M^{2}}\right)\right],
$$

which can then be inserted in (2.20) to obtain an exact, if somewhat unwieldy, formula for the maximal volume as a function of $t_{R}+t_{L}$.

The expression for the volume simplifies enormously at late times,

$$
V=\frac{M}{2}\left(t_{R}+t_{L}\right)+e^{2 \sigma_{a}}+\frac{M}{2}\left(1+2 \sigma_{a}-\log \frac{M}{2}\right)+\mathcal{O}\left(e^{-2\left(t_{R}+t_{L}\right)}\right),
$$

with a leading term that grows linearly with time, followed by a constant term that depends on the location of the anchor curve, and subsequent terms that are exponentially suppressed at late times. As expected, the volume diverges in the $\sigma_{a} \rightarrow \infty$ limit, where the anchor curves are moved off to spatial infinity, but the late time rate of growth is unaffected by the location of the anchor curves. The volume prescription for complexity is sometimes taken to only include the volume inside the event horizon of the black hole. This amounts to cutting off the volume integration in (2.20) at the event horizon,

$$
\begin{aligned}
V_{\mathrm{EH}} & =\epsilon \int_{0}^{\mu} d \tau \cosh \tau \cosh (\tau-\mu) \\
& =\frac{M}{2}(\mu+\tanh \mu)
\end{aligned}
$$

which can be shown to grow at the same rate at late times as the full volume between anchor curves. Later on, when we consider dynamical black holes formed by the gravitational collapse of matter, we will see that the stretched horizon is a natural choice of anchor curve. In the case at hand, we define the stretched horizon to be a membrane outside the black hole, with an area that is one unit larger than the area of the event horizon,

$$
e^{-2 \phi_{\mathrm{SH}}}=e^{-2 \phi_{\mathrm{EH}}}+1=M+1 .
$$


This is a curve of constant dilaton field outside the black hole, which is how we defined our anchor curves above. Indeed, with this definition, the stretched horizon is located at $\sigma_{\mathrm{SH}}=0$ in the $(t, \sigma)$ coordinate system and the volume inside the stretched horizon can be obtained by setting $\sigma_{a}=0$ in (2.22). The result differs from the volume inside the event horizon by only a small amount and the late time volume growth is the same. For a static two-sided black hole the location of the anchor curve is unimportant if all we are interested in is the late time rate of growth of the complexity. It is only when we consider dynamical black holes that the advantage of using the stretched horizon as the anchor curve becomes apparent.

We note that the Hawking temperature of a CGHS black hole is $T_{\mathrm{H}}=\frac{1}{2 \pi}$, independent of the black hole mass [7], and the Bekenstein-Hawking entropy is given by $S_{\mathrm{BH}}=2 M$. The late time rate of growth of the complexity given by the volume in (2.22) is thus proportional to $S_{\mathrm{BH}} T_{\mathrm{H}}$, which is precisely in line with the original $\mathcal{C}=V$ proposal [3].

\subsection{Complexity of a black hole formed by gravitational collapse}

Next, we consider collapsing a thin shell of matter at $x^{+}=x_{0}^{+}$, mediated by matter fields $f_{i}$, into a CGHS vacuum and creating a black hole. This amounts to

$$
T_{++}^{f}=\frac{M}{x_{0}^{+}} \delta\left(x^{+}-x_{0}^{+}\right)
$$

where $\delta\left(x^{+}-x_{0}^{+}\right)$is a delta function. For the geometry this implies

$$
e^{-2 \phi}=e^{-2 \rho}= \begin{cases}-x^{+} x^{-} & \text {if } x^{+}<x_{0}^{+}, \\ -x^{+}\left(x^{-}+\frac{M}{x_{0}^{+}}\right)+M & \text { if } x^{+} \geq x_{0}^{+} .\end{cases}
$$

The resulting black hole is one-sided, as illustrated in figure 2 .

Just as in the eternal black hole geometry, we need to introduce an anchor curve outside the black hole to obtain a finite volume. The extremal curve now reaches from a point on the anchor curve to a point on $x^{-} x^{+}=0$. In figure 2, we sketch the setup. In contrast to the eternal black hole where we had two anchor points and therefore a unique extremal curve connecting these two points, there is now only one anchor point and therefore we have to supply a prescription for additional boundary conditions. In [16], for instance, it was argued that in order to obtain a smooth volume at the radial origin, the additional boundary conditions should be $t^{\prime}(r)=0$ at $r=0$. Expressing the corresponding condition in our setup in Kruskal coordinates one finds the relation

$$
x^{-} \dot{x}^{+}-x^{+} \dot{x}^{-}=0
$$

at $x^{+} x^{-}=0$. An alternative prescription is to consider all locally extremal curves originating from the anchor point, and selecting the curve that maximizes the volume inside the black hole. This computation can be done and, interestingly, it turns out that this prescription leads to exactly the same curves as the boundary conditions (2.27).

Curves that maximise the volume functional (2.4) in the one-sided black hole background are obtained by patching together maximal curves across the infalling shockwave. 

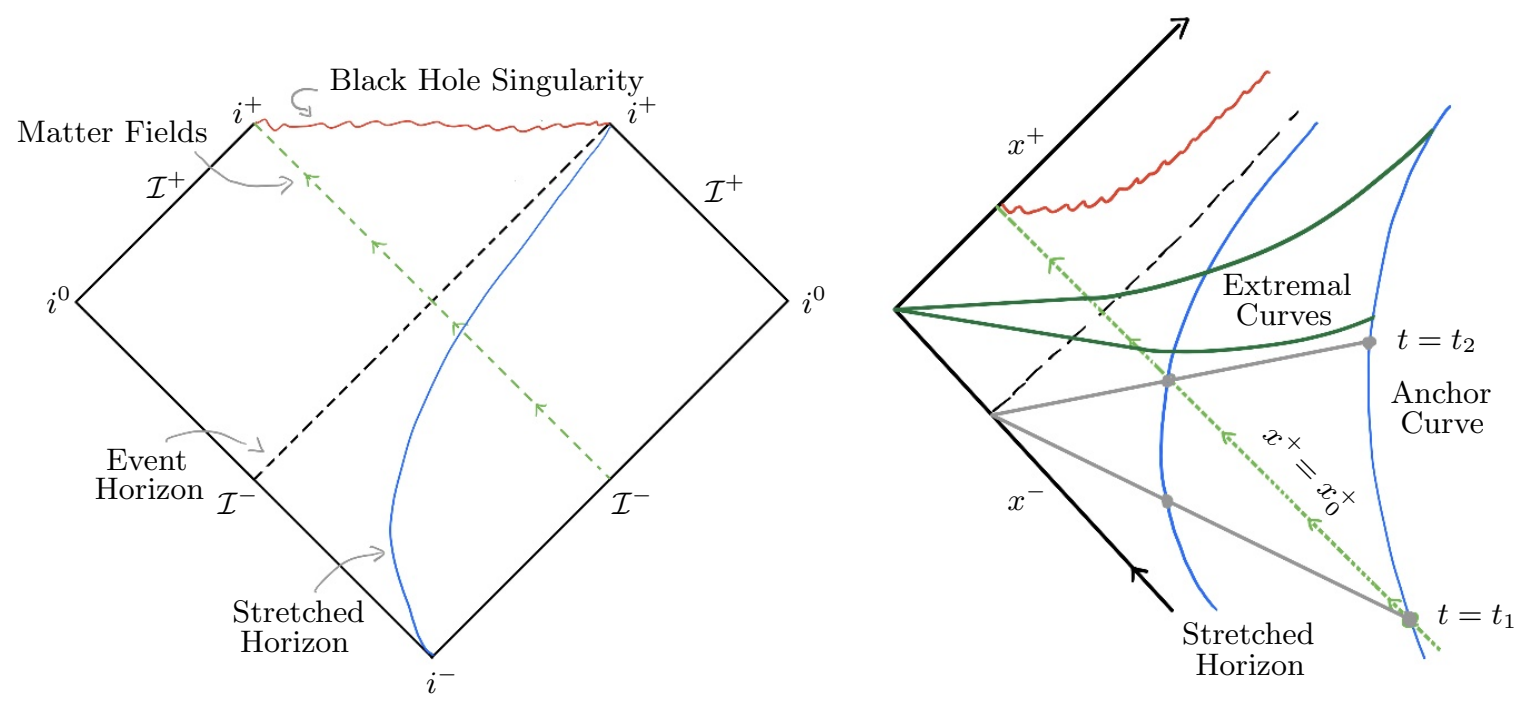

Figure 2. (Left) This cartoon depicts the Penrose diagram of a one-sided CGHS black hole formed by gravitational collapse. (Right) This is a Kruskal diagram of the Penrose diagram on the left. The color coding coincides. The gray line is an equal time curve.

In the inside region, $0<x^{+}<x_{0}^{+}$, the geometry is flat and the volume functional takes the simple form,

$$
V=\int d s \sqrt{y^{+} \dot{y}^{+} y^{-} \dot{y}^{-}}
$$

One obtains a two-parameter family of maximal curves,

$$
\left(x^{+}\right)^{2}=\alpha\left(x^{-}\right)^{2}+\beta
$$

where $\alpha>0$ and $\beta<\sqrt{x_{0}^{-}}$are real valued parameters. The boundary condition (2.27) selects curves with $\beta=0$, which are simply straight lines emanating from the origin $x^{+}=x^{-}=0$ in Kruskal coordinates (see figure 2).

In the outside region, $x^{+}>x_{0}^{+}$, the geometry is that of a static black hole and the volume functional reduces to (2.8) in shifted Kruskal coordinates,

$$
y^{+}=x^{+}, \quad y^{-}=x^{-}+\frac{M}{x_{0}^{+}} .
$$

There is again a two-parameter family of maximal volume curves satisfying (2.13) and labelled by $E$ and $t_{0}$. We use Weierstraß-Erdmann conditions to patch across the shockwave. First of all, the curve itself should be continuous. A second condition comes from viewing the integral in the volume functional as a Lagrange density and requiring that the momenta conjugate to $y^{+}$and $y^{-}$be continuous across the shock. Those matching conditions uniquely determine the parameter $\alpha$ and $\beta$ in terms of $E$ and $t_{0}$, and vice versa. One finds, in particular, that curves with $\beta=0$ in the inside region match onto curves with $E=M$ on the outside leaving us with a one-parameter family

$$
y^{-}= \begin{cases}\frac{M}{2 y^{+}}-\frac{e^{-2 t_{0}}}{4 M} y^{+} & y^{+}>x_{0}^{+}, \\ \frac{M}{x_{0}^{+}}-\left(\frac{M}{2 x_{0}^{+}}+\frac{e^{-2 t_{0}}}{4 M}\right) y^{+} & y^{+}<x_{0}^{+} .\end{cases}
$$


Subsequently, the remaining parameter $t_{0}$ can be uniquely related to the tortoise time $t$ on the anchor curve, see figure 2. As in section 2.1, we define the anchor curves, parametrised by $\sigma_{a}$, so that the dilaton field is constant $\phi=\phi_{a}$,

$$
e^{2 \sigma_{a}}=e^{-2 \phi_{a}}-M=\left\{\begin{array}{ll}
-y^{+} y^{-} & y^{+}>x_{0}^{+} \\
-y^{+}\left(y^{-}-\frac{M}{x_{0}^{+}}\right)-M & y^{+}<x_{0}^{+}
\end{array} .\right.
$$

We now obtain a relation between $\sigma_{a}, t_{0}$ and $t$ by combining (2.31) and (2.32). One is now in a position to evaluate the volume functional (2.4).

The growth of the volume inside the stretched horizon as a function of tortoise time $t$ is given by

$$
V_{\mathrm{SH}}^{\prime}(t)= \begin{cases}\frac{1}{2} M & t \geq t_{2}, \\ \frac{1}{2} M \cosh (t)^{-2}+\mathcal{O}\left(\frac{1}{M}\right) & t<t_{2},\end{cases}
$$

where $t_{2}$ is the moment the black hole is formed. We conclude that the volume growth sets in essentially at the time the black hole is formed, which is consistent with causality.

In contrast to the aforementioned, we could consider the total volume up to an arbitrary anchor line. ${ }^{1}$ The result agrees at times $t>t_{2}$ but disagrees strongly before that time. We have

$$
V_{\mathrm{AC}}^{\prime}(t)= \begin{cases}\frac{1}{2} M & t \geq t_{1}, \\ 0 & t<t_{1},\end{cases}
$$

where $t_{1}$ is the time when the anchor curve crosses the shockwave line, see figure 2 . This is long before the black hole is created ${ }^{2}$ and it would imply that complexity starts growing at the moment the shockwave is released, see figure 3. For this reason, we prefer to use the stretched horizon as the anchor curve and only consider the volume inside the black hole. The volume integral can be cut off at either the event horizon or at the stretched horizon.

We can compare the complexity growth of the gravitational collapse model to the complexity growth of the eternal black hole, both at late time. The result for the gravitational collapse model is $V^{\prime}=M / 2$ for $t>t_{2}$, which is exactly half of the eternal black hole result, see (2.23). This factor $\frac{1}{2}$ corresponds to the fact that in the current case we only consider half of the volume slice (since we only have a one-sided black hole), as compared to the eternal black hole case (which is two-sided).

\section{The RST model: complexity in a semi-classical black hole}

The CGHS model can be extended such that one can study a semi-classical black hole analytically [17, 18]. Here we adopt a particular modification due to RST [8], which is

\footnotetext{
${ }^{1}$ In particular, we could imagine this anchor line to be asymptotically far away, in analogy to computations done in the AdS/CFT setup.

${ }^{2}$ In fact, as we move the anchor curve infinitely far away, the time difference of black hole creation and $t_{R}^{1}$ also goes to infinity.
} 


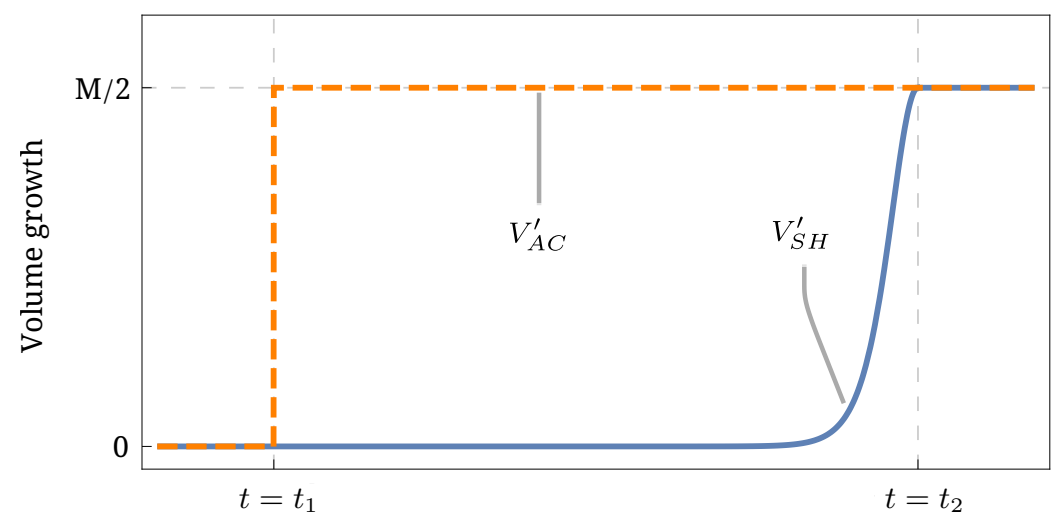

Time $t$

Figure 3. This graph demonstrates the difference between the volume growth $V_{\mathrm{SH}}^{\prime}$ and $V_{\mathrm{AC}}^{\prime} \cdot V_{\mathrm{AC}}^{\prime}$ starts growing at the time the shockwave crosses the anchor curve $t_{1}$ which is much earlier than the time $t_{2}$ the black hole is created.

given by

$$
S_{\mathrm{RST}}=S_{\mathrm{CGHS}}+S_{\mathrm{q}}+S_{\mathrm{ct}} .
$$

If one takes $N$, the number of matter fields $f_{i}$, to be large, then $S_{\mathrm{q}}$ represents the leading order quantum correction due to matter, which in conformal gauge reads

$$
S_{q}=-\kappa \int_{\mathcal{M}} d^{2} x \partial_{+} \rho \partial_{-} \rho
$$

Here $\kappa:=N / 12$ can be thought of as playing the role of $\hbar$, which is put to unity. The additional RST counter term has the following form in conformal gauge,

$$
S_{\mathrm{ct}}=-\kappa \int_{\mathcal{M}} d^{2} x \phi \partial_{+} \partial_{-} \rho
$$

This term is allowed by the symmetries of the model and when it is added the semi-classical field equations take a particularly simple form and are easily solved analytically.

The solutions of the equations of motion can be written in compact form if one defines new field variables,

$$
\sqrt{\kappa} \Omega:=e^{-2 \phi}+\frac{\kappa}{2} \phi, \quad \sqrt{\kappa} \chi:=e^{-2 \phi}-\frac{\kappa}{2} \phi+\kappa \rho .
$$

Using the new fields, the semi-classical action reads

$$
S_{\mathrm{RST}}=2 \int_{\mathcal{M}} d^{2} x\left[-\partial_{+} \chi \partial_{-} \chi+\partial_{+} \Omega \partial_{-} \Omega+e^{\frac{2}{\sqrt{k}}(\chi-\Omega)}+\frac{1}{2} \sum_{i=1}^{N} \partial_{+} f_{i} \partial_{-} f_{i}\right] .
$$

The RST model continues to enjoy the symmetry of the classical theory that allowed us to choose Kruskal coordinates, setting $\phi=\rho$ and as a result $\Omega=\chi$.

Again, we study an incoming shockwave of energy $M$ at $x^{+}=x_{0}^{+}$of the form

$$
T_{++}^{f}=\frac{M}{x_{0}^{+}} \delta\left(x^{+}-x_{0}^{+}\right) .
$$



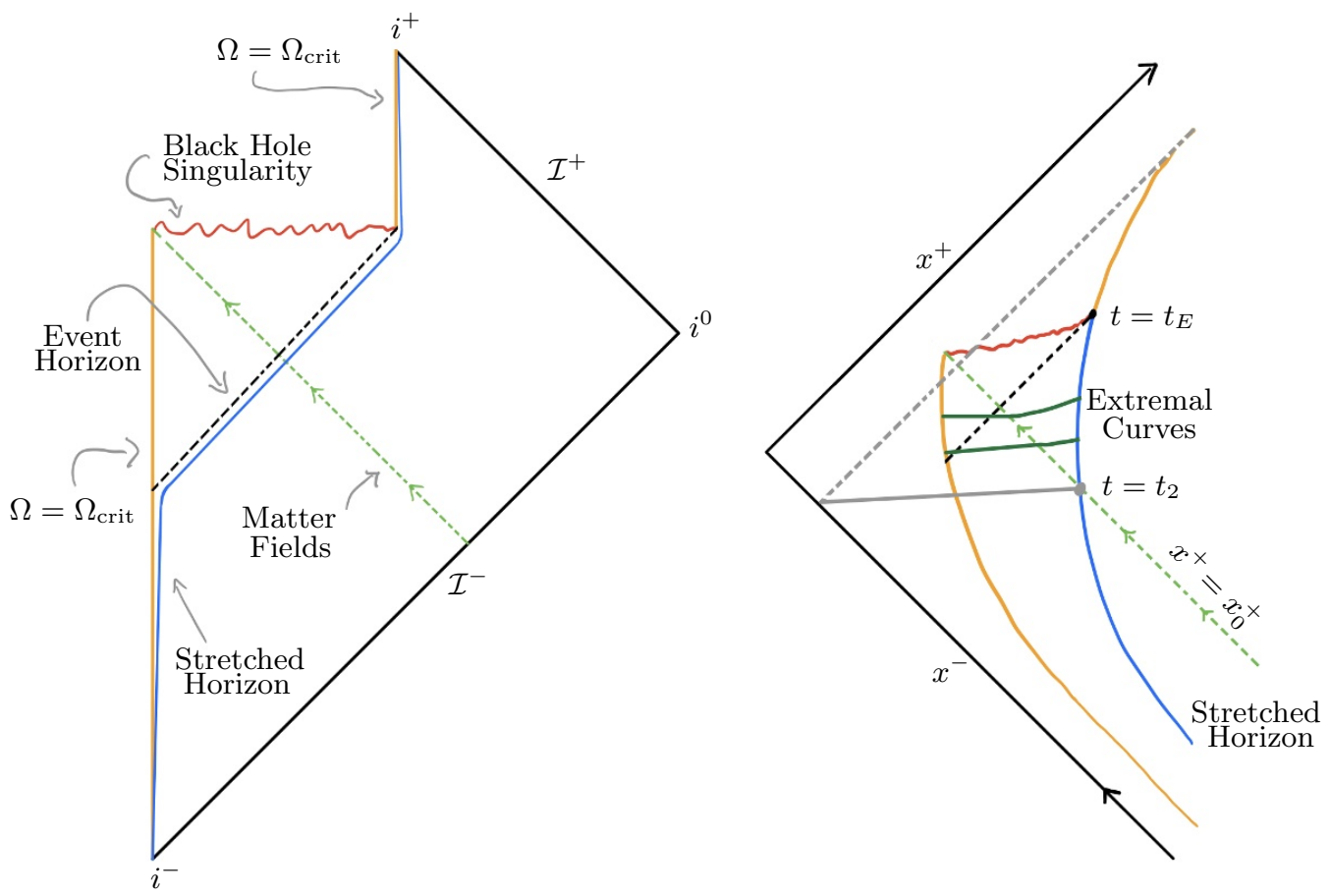

Figure 4. (Left) Cartoon of the Penrose diagram of the life cycle of an evaporating black hole formed by collapse. (Right) Depicts Kruskal diagram of the scenario illustrated in the left figure. The color coding coincides.

The semi-classical collapse solution of interest, given in Kruskal coordinates, is

$$
\sqrt{\kappa} \Omega\left(x^{+}, x^{-}\right)=-x^{+} x^{-}+\left(x_{0}^{+}-x^{+}\right) \frac{M}{x_{0}^{+}} \Theta\left(x^{+}-x_{0}^{+}\right)-\frac{\kappa}{4} \ln \left(-x^{+} x^{-}\right) .
$$

The solution describes flat spacetime for $x^{+} \leq x_{0}^{+}$and an evaporating black hole for $x^{+}>x_{0}^{+}$, as shown in figure 4. It is important to note that in the RST model only those regions of spacetime where $\Omega\left(x^{+}, x^{-}\right) \geq \Omega_{\text {crit }}:=\frac{\sqrt{\kappa}}{4}\left(1-\log \left(\frac{\kappa}{4}\right)\right)$ are considered physical. In the flat spacetime region inside the infalling shell, the boundary of the physical region is a timelike curve that can be interpreted as the origin in spherical coordinates in a higherdimensional parent theory. For $x^{+}>x_{0}^{+}$, the curve $\left(x_{S}^{+}, x_{S}^{-}\right)$for which $\Omega\left(x_{S}^{+}, x_{S}^{-}\right)=\Omega_{\text {crit }}$ turns spacelike and defines the location of the black hole singularity. The semi-classical black hole evaporates and eventually the singularity terminates at an endpoint, after which the solution can be extended into a late time flat region where the physical boundary is again timelike. A more detailed description of the semi-classical geometry can for instance be found in [12].

Although we have closed expressions for the solutions of the RST model, we were not able to solve the extremization problem of a spacelike volume analytically and in order to check whether the complexity as volume prescription gives results consistent with general expectations we had to resort to numerical methods.

Since the extremization of

$$
V=\int d s e^{-2 \phi} \sqrt{g_{\mu \nu} \dot{y}^{\mu} \dot{y}^{\nu}}
$$


can be performed by solving corresponding Euler-Lagrange equations, the numerical problem is simply to solve a non-linear ordinary differential equation with appropriate boundary conditions, whose solutions provide a parametrisation of the extremized volume. Numerically integrating (3.8) inside the stretched horizon provides us with the volume complexity $V(t)$ at a value of tortoise time $t$ that is determined by the choice of anchor curve in the same way as for a classical dynamical black hole. If we use a curve of constant $\Omega$ far from the black hole, then the complexity begins to grow very early, long before the incoming shockwave reaches the stretched horizon. If we instead use the stretched horizon as our anchor curve, then the complexity growth turns on essentially when the black hole is formed and this prescription is used in obtaining the numerical results presented in figure 5 . We note, however, that the choice of anchor curve only affects the onset time and the early growth rate of the complexity, but not the slope of the curve (at leading order for large $M / \kappa)$ showing the decreasing growth rate after a scrambling time has passed from the onset of complexity growth.

Note that, like in the case of classical collapse, it is not a priori clear what the appropriate boundary conditions at the origin are. We have chosen to apply boundary conditions analogous to (2.27) at $\Omega=\Omega_{\text {crit }}$ and then use a shooting algorithm to obtain the corresponding maximal volume curve. We apply the Weierstraß-Erdmann matching conditions to patch across the shockwave and then continue the numerical evaluation outwards.

Further, it could be reasoned that the factor $e^{-2 \phi}$ in (3.8), which is interpreted as the area of the transverse two-sphere in the higher-dimensional theory, should be replaced by the quantum corrected area $\Omega-\Omega_{\text {crit }}$. We find that the slope of $V^{\prime}(t)$ is not particularly sensitive to this replacement, at least not in the parameter range where our numerical evaluation is reliable (see below).

Results for the functions $V^{\prime}(t)$ for different values of $M / \kappa$ are plotted in figure 5 . We observe that for reasonably high values of $M / \kappa$ the numerical result is consistent with a linear decrease after the scrambling time $t_{S}=\log (4 M / \kappa)$. We do not expect that the volume growth follows the linear trend forever, since eventually, the black hole is small enough, so that quantum corrections become strong on the stretched horizon. In this model, the coupling strength ${ }^{3}$ on the stretched horizon at the scrambling time is approximately given by $\left(M-\frac{\kappa}{4} t_{S}\right)^{-\frac{1}{2}}$. For instance, a ratio $M / \kappa=1$ yields a coupling strength of $\approx 1.2$ which indicates we should not trust the result at all for this choice of parameters. This is reflected in figure $5 \mathrm{a}$ which barely exhibits a linear growth rate. On the other hand a ratio $M / \kappa=100$ yields a coupling strength of $\approx 0.1$ which demonstrates that we should be able to trust the solution for quite some time after the scrambling time. This is confirmed in figure $5 \mathrm{c}$ and $5 \mathrm{~d}$ which shows a linearly falling growth rate over a long period of time.

\section{Conclusion and outlook}

In this paper we have computed holographic complexity as the volume of Einstein-Rosen bridges inside black holes in two-dimensional dilaton gravity models where we have explic-

\footnotetext{
${ }^{3}$ In this model the gravitational coupling is given by the value of $\left(e^{-2 \phi}-\frac{\kappa}{4}\right)^{-\frac{1}{2}}$.
} 


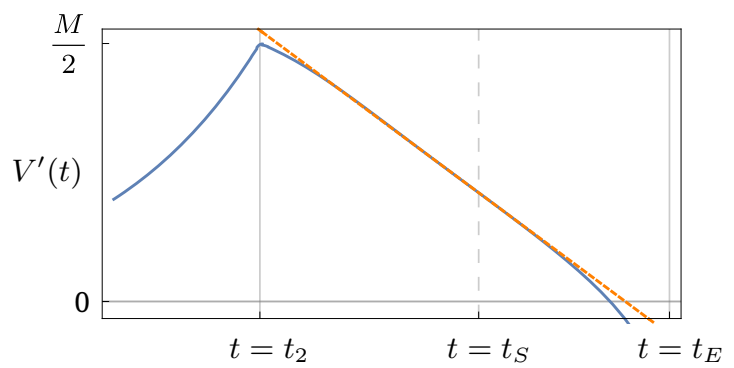

(a) $M / \kappa=1$.

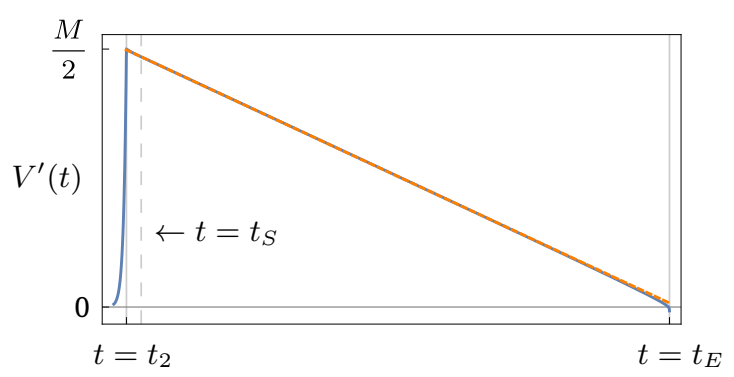

(c) $M / \kappa=50$.

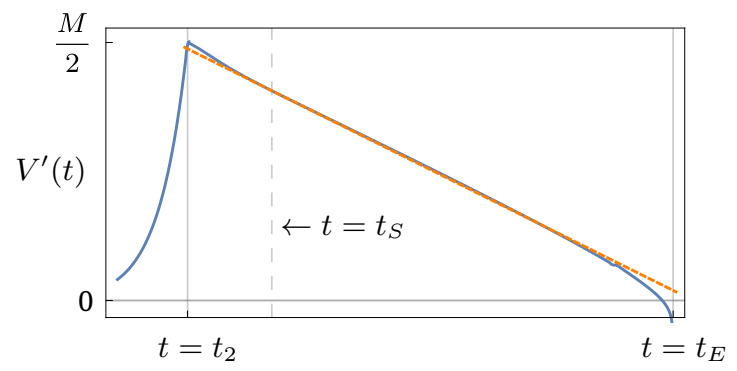

(b) $M / \kappa=5$.

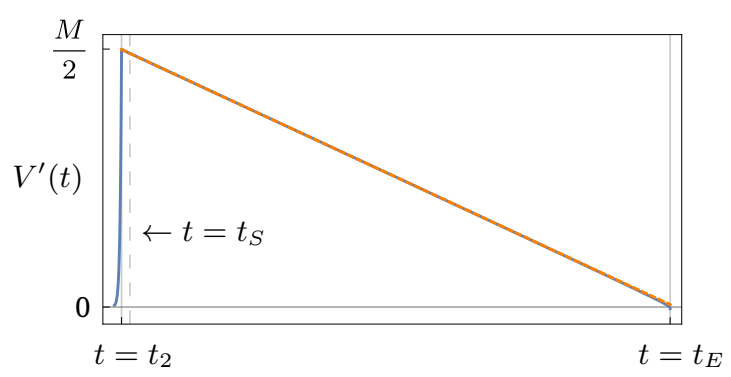

(d) $M / \kappa=100$.

Figure 5. Numerical results of volume growth for different values of $M / \kappa$. The black hole creation time is indicated by $t=t_{2}$ while the evaporation process is completed at time $t=t_{E}$. The blue curve depicts the numerical result while the dashed orange line is obtained by a linear extrapolation of the curve around the scrambling time $t_{S}$.

itly known semi-classical black hole solutions. This allows us to follow the time evolution of the complexity of a black hole that is formed in gravitational collapse and subsequently evaporates by emitting Hawking radiation. Our main results can be summarized in three statements.

First, in order to obtain sensible results, we have to calculate extremal volumes in the four-dimensional parent theory rather than lengths of geodesics in the two-dimensional reduction. The appropriately defined volume functional can be explicitly evaluated in the classical CGHS model and it exhibits the expected linear growth with Schwarzschild time at late times. At the same time it is easy to check that the length of the spacelike geodesics, that will otherwise arise, does not lend itself to a direct interpretation in terms of complexity.

Second, when considering dynamical black holes formed by gravitational collapse, we find it natural to cut off the volume integration at the stretched horizon of the black hole rather than extending the integration range to a distant anchor curve. This distinction is unimportant if all we are interested in is the late time rate of growth of the complexity for a classical black hole but it does affect the onset of complexity growth. If the volume prescription extends to a distant anchor curve then the complexity already starts growing as soon the infalling shockwave passes the anchor point and the complexity growth turns on abruptly. If, on the other hand, we use the stretched horizon to delimit the integration range, then complexity growth turns on smoothly at a time that coincides with the onset of Hawking emission at the semi-classical level. 
Third, using numerical methods, we obtain the complexity of an evaporating black hole as a function of time using the volume prescription inside the stretched horizon. We find that after the black hole is created, the complexity growth needs a time period of order the scrambling time to settle to a rate of growth proportional to the area of the stretched horizon. The growth rate then reliably tracks the area of the horizon as it shrinks due to black hole evaporation. Towards the end of the black hole lifetime, higher order quantum corrections are expected to become important and semi-classical calculations can no longer be trusted.

Holographic complexity can also be calculated in these models using the WheelerDeWitt action formalism. We will present our results on that in a forthcoming companion paper [6], where we find that the numerical results obtained in the present paper for the volume complexity are confirmed by action calculations that can be carried out analytically even at the semi-classical level.

Our results fit very well with Susskind's argument [2], that the rate of complexity growth for an evaporating black hole should at any given time be proportional to the product of the black hole entropy $S$ and the Hawking temperature $T$ at that time. In the models we are considering, the Hawking temperature remains constant and the entropy is proportional to black hole mass. It follows that the black hole loses area at a constant rate determined by $N$ the number of matter channels available for Hawking emission. This translates into a growth rate of complexity that is initially proportional to the initial mass of the black hole and then drops linearly with time until the black hole has completely evaporated. In other words, $\dot{\mathcal{C}} \propto S T$ should decrease linearly in time. This precisely the behaviour we see in our numerical calculations, following an initial onset period of order the scrambling time, which is logarithmic in $M$ in these models.

Our results also support the notion that the holographic complexity corresponds to the quantum complexity of the combined system of black hole and emitted Hawking radiation [2]. The scrambling dynamics that generates the growth in complexity takes place at the stretched horizon and the growth rate is reduced as the area of the horizon shrinks. The outgoing radiation is free streaming and no further complexity is generated by the degrees of freedom that have been emitted from the black hole. At the end of the day, the black hole has disappeared and all that is left is a long train of outgoing radiation in a state of high, but no longer growing, complexity.

\section{Acknowledgments}

It is a pleasure to thank Shira Chapman and Nick Poovuttikul for discussions. This research was supported by the Icelandic Research Fund under grants 185371-051 and 195970-051, and by the University of Iceland Research Fund.

Open Access. This article is distributed under the terms of the Creative Commons Attribution License (CC-BY 4.0), which permits any use, distribution and reproduction in any medium, provided the original author(s) and source are credited. 


\section{References}

[1] L. Susskind, L. Thorlacius and J. Uglum, The Stretched horizon and black hole complementarity, Phys. Rev. D 48 (1993) 3743 [hep-th/9306069] [INSPIRE].

[2] L. Susskind, Three Lectures on Complexity and Black Holes, arXiv:1810.11563 [INSPIRE].

[3] L. Susskind, Computational Complexity and Black Hole Horizons, Fortsch. Phys. 64 (2016) 44 [arXiv: 1403.5695] [INSPIRE].

[4] A.R. Brown, D.A. Roberts, L. Susskind, B. Swingle and Y. Zhao, Holographic Complexity Equals Bulk Action?, Phys. Rev. Lett. 116 (2016) 191301 [arXiv:1509.07876] [INSPIRE].

[5] A.R. Brown, D.A. Roberts, L. Susskind, B. Swingle and Y. Zhao, Complexity, action and black holes, Phys. Rev. D 93 (2016) 086006 [arXiv:1512.04993] [InSPIRE].

[6] L. Schneiderbauer, W. Sybesma and L. Thorlacius, Action Complexity for Semi-Classical Black Holes, arXiv:2001.06453 [INSPIRE].

[7] C.G. Callan Jr., S.B. Giddings, J.A. Harvey and A. Strominger, Evanescent black holes, Phys. Rev. D 45 (1992) R1005 [hep-th/9111056] [InSPIRE].

[8] J.G. Russo, L. Susskind and L. Thorlacius, Black hole evaporation in $(1+1)$-dimensions, Phys. Lett. B 292 (1992) 13 [hep-th/9201074] [INSPIRE].

[9] S.B. Giddings and A. Strominger, Dynamics of extremal black holes, Phys. Rev. D 46 (1992) 627 [hep-th/9202004] [INSPIRE].

[10] T. Banks, A. Dabholkar, M.R. Douglas and M. O'Loughlin, Are horned particles the climax of Hawking evaporation?, Phys. Rev. D 45 (1992) 3607 [hep-th/9201061] [INSPIRE].

[11] J.A. Harvey and A. Strominger, Quantum aspects of black holes, in proceedings of the Theoretical Advanced Study Institute (TASI 92): From Black Holes and Strings to Particles, Boulder, U.S.A., 1-26 June 1992, pp. 537-588, [hep-th/9209055] [INSPIRE].

[12] L. Thorlacius, Black hole evolution, Nucl. Phys. Proc. Suppl. 41 (1995) 245 [hep-th/9411020] [INSPIRE].

[13] A. Strominger, Les Houches lectures on black holes, in proceedings of the NATO Advanced Study Institute: Les Houches Summer School, Session 62: Fluctuating Geometries in Statistical Mechanics and Field Theory, Les Houches, France, 2 August-9 September 1994, hep-th/9501071 [INSPIRE].

[14] S.B. Giddings, Quantum mechanics of black holes, in proceedings of the Summer School in High-energy physics and cosmology, Trieste, Italy, 13 June-29 July 1994, pp. 0530-574 [hep-th/9412138] [INSPIRE].

[15] A.R. Brown, H. Gharibyan, H.W. Lin, L. Susskind, L. Thorlacius and Y. Zhao, Complexity of Jackiw-Teitelboim gravity, Phys. Rev. D 99 (2019) 046016 [arXiv:1810.08741] [INSPIRE].

[16] S. Chapman, H. Marrochio and R.C. Myers, Holographic complexity in Vaidya spacetimes. Part I, JHEP 06 (2018) 046 [arXiv: 1804.07410] [INSPIRE].

[17] A. Bilal and C.G. Callan Jr., Liouville models of black hole evaporation, Nucl. Phys. B 394 (1993) 73 [hep-th/9205089] [INSPIRE].

[18] S.P. de Alwis, Quantization of a theory of $2 D$ dilaton gravity, Phys. Lett. B 289 (1992) 278 [hep-th/9205069] [INSPIRE]. 